\title{
Spherically Symmetric Similarity Solutions of the Einstein Field Equations for a Perfect Fluid ${ }^{\star}$
}

\author{
M. E. CAHILl and A. H. TAub \\ Mathematics Department, University of California, Berkeley, California
}

Received November 30, 1970

\begin{abstract}
Spherically symmetric space-times which admit a one parameter group of conformal transformations generated by a vector $\xi^{\mu}$ such that $\xi_{\mu ; \nu}+\xi_{v ; \mu}=2 g_{\mu \nu}$ are studied. It is shown that the metric coefficients of such space-times depend essentially on the single variable $z=r / t$ where $r$ is a radial coordinate and $t$ is the time. The Einstein field equations then reduce to ordinary differential equations. The solutions of these equations are analogous to the similarity solutions of the classical theory of hydrodynamics. In case the source of the field is a perfect fluid whose specific internal energy is a function of temperature alone, the solution of the field equations is uniquely determined by specifying data on the time-like hypersurface $z=$ constant and is a similarity solution. The problem of fitting a similarity solution to another solution of the field equations across a shock described by the hypersurface $z=$ constant is treated. A particular similarity solution for which $w=3 p$ obtains is shown to describe a Robertson-Walker space-time. This solution is fitted to a special static solution of the Einstein field equations which has a singularity at $r=0$. The resulting solution of the Einstein field equations is shown to be regular everywhere except at $r=0 \geqq t$ and the shock. The special Robertson-Walker metric is also fitted to a particular class of collapsing dust solutions (which are also similarity solutions) across a shock. The resulting solution is regular everywhere except at $r=t=0$ and on the shock.
\end{abstract}

\section{Introduction}

In non-relativistic continuum mechanics there is a classical procedure for reducing the partial differential equations which characterize a given problem involving high symmetry to ordinary ones. This consists in assuming a specific form for the solution in which the dependent variables are taken to be essentially functions of a single independent variable. This variable is a dimensionless combination of the independent variables, namely the space coordinates and the time. Thus in a spherically symmetric problem where the independent variables are a distance from the center of symmetry, $r$, and the time $t$, the dependent variables are

* This work was supported in part by the United States Atomic Energy Commission under contract number AT(04-3)-34 Project Agreement No. 125. It was completed when one of the authors (A.H.T.) was on sabbatical leave from the University of California, Berkeley and in residence at the Department of Applied Mathematics and Theoretical Physics, Cambridge University. 
assumed to be essentially functions of the variable

$$
z=r / r_{0}\left(t / t_{0}\right)^{-n} .
$$

Such solutions are called similarity solutions or progressive waves (cf. [1]). von Neumann [2] and Taylor [3] have applied this technique to the determination of the flow behind a strong shock wave created by a point source from which a finite amount of energy was released, that is, an explosion. Kopal and his coworkers [4] have given solutions for similar problems in the non-relativistic theory of self-gravitating fluids.

It is the purpose of this paper to characterize and discuss similarity solutions to the Einstein field equations for a spherically symmetric distribution of a self-gravitating perfect fluid. We shall assume that the fluid is described by a caloric equation of state which may be expressed by the equation

$$
w+p=c^{2} \varrho G(x),
$$

where $w$ is the rest energy density, $p$ is the pressure, $\varrho$ is the rest mass density, $c$ is the special relativistic velocity of light and

$$
p x=\varrho c^{2} .
$$

It can be shown [5] that $x$ is proportional to the inverse of the temperature. Different fluids are described by different functions $G$. It will also be assumed that rest mass is conserved.

We shall also discuss similarity solutions behind shock waves. That is, we shall assume that we are dealing with two regions of space-time separated by a hypersurface with a space-like normal vector. Across this hypersurface which represents the shock front the generalised RankineHugoniot equations are required to hold. In addition the first and second fundamental forms of the hypersurface are required to be continuous. In one region of space-time bounded by this hypersurface, a similarity solution of the field equations will be assumed to obtain. We shall examine the nature of the solutions of the field equations that can be fitted to a similarity solution across a shock front.

We shall use comoving coordinates in both regions of space-time. In these the metric may be written as

$$
d s^{2}=e^{2 \varphi} d t^{2}-\frac{e^{2 \psi}}{c^{2}} d r^{2}-\frac{R^{2}}{c^{2}} d \Omega^{2}
$$

where $\varphi, \psi$ and $R$ are functions of $r$ and $t$, and

$$
d \Omega^{2}=d \theta^{2}+\sin ^{2} \theta d \chi^{2}
$$


$\theta$ and $\chi$ labelling points on the unit sphere. In this coordinate system the field equations

become

$$
G_{v}^{\mu}=-\kappa T_{v}^{\mu}=-\kappa\left[(w+p) u^{\mu} u_{v}-p \delta_{v}^{\mu}\right],
$$

$$
\begin{aligned}
& c^{2} \mathrm{e}^{\varphi+\psi}+\mathrm{e}^{\psi-\varphi}\left(R_{t}^{2}+2 R R_{t} \psi_{t}\right)-c^{2} \mathrm{e}^{\varphi-\psi}\left[2 R R_{r r}+R_{r}^{2}-2 R R_{r} \psi_{r}\right] \\
& =\kappa w R^{2} \mathrm{e}^{\varphi+\psi} \text {, } \\
& c^{2} \mathrm{e}^{\varphi+\psi}+\mathrm{e}^{\psi-\varphi}\left(2 R R_{t t}+R_{t}^{2}-2 R R_{t} \varphi_{t}\right)-c^{2} \mathrm{e}^{\varphi-\psi}\left(R_{r}^{2}+2 R R_{r} \varphi_{r}\right) \\
& =-\kappa p R^{2} \mathrm{e}^{\varphi+\psi} \text {, } \\
& \mathrm{e}^{\psi-\varphi}\left(\frac{R_{t t}}{R}+\psi_{t t}+\psi_{t}^{2}+\frac{R_{t}}{R} \psi_{t}-\frac{R_{t}}{R} \varphi_{t}-\psi_{t} \varphi_{t}\right) \\
& -c^{2} \mathrm{e}^{\varphi-\psi}\left(\frac{R_{r r}}{R}+\varphi_{r r}+\varphi_{r}^{2}+\frac{R_{r}}{R} \varphi_{r}-\frac{R_{r}}{R} \psi_{r}-\varphi_{r} \psi_{r}\right)=-\kappa p \mathrm{e}^{\varphi+\psi}
\end{aligned}
$$

and

$$
R_{r t}-R_{t} \varphi_{r}-R_{r} \psi_{t}=0
$$

with $\kappa=8 \pi G / c^{2}$. Eq. (1.6) is the statement that the coordinate system is a comoving one, that is

$$
u^{\mu}=\mathrm{e}^{-\varphi} \delta_{4}^{\mu} .
$$

The Bianchi identities imply that

and

$$
\varphi_{r}=-\frac{p_{r}}{w+p}
$$

$$
\psi_{t}=-\frac{w_{t}}{w+p}-\frac{2 R_{t}}{R} .
$$

When we supplement the field equations by the equation of conservation of mass, that is the equation

then we also have

$$
\left(\varrho u^{\mu}\right)_{; \mu}=0
$$

$$
\psi_{t}=-\frac{\varrho_{t}}{\varrho}-\frac{2 R_{t}}{R} .
$$

Eqs. (1.9) and (1.11) imply that

$$
S_{t}=0
$$

where $S$ is the specific rest entropy and is defined as

$$
\theta d S=d\left(\frac{w+p}{\varrho}\right)-\frac{1}{\varrho} d p
$$


with $\theta$ the temperature. The latter quantity is defined in terms of $p$ and $\varrho$ as the integrating factor of Eq. (1.13). This equation may be expressed in terms of the function $G(x)$ introduced above and becomes

$$
\frac{x \theta}{c^{2}} d S=-\left(\frac{1}{L} \frac{d L}{d x} d x+\frac{d \varrho}{\varrho}\right)
$$

where

$$
\frac{1}{L} \frac{d L}{d x}=-\left(x \frac{d G}{d x}+\frac{1}{x}\right)
$$

Hence

$$
x \theta=c^{2} C
$$

where $C$ is a constant and

$$
\mathrm{e}^{-C\left(S-S_{0}\right)}=\varrho L(x)=\frac{p}{c^{2}} x L(x),
$$

with $S_{0}$ a constant of integration.

Eq. (1.15) relates the temperature $\theta$ to $x$ and Eq. (1.16) expresses the specific entropy as a function of two independent thermodynamic variables. Eq. (1.12) may now be written as

$$
p=\frac{K(r)}{x L(x)}
$$

where $K(r)$ may be an arbitrary function of its argument. For isentropic flows it is a constant.

The function $\psi$ may be expressed in terms of $L(x)$ for it follows from Eq. (1.11) that

$$
f(r) \mathrm{e}^{-\psi}=c^{2} \varrho R^{2}
$$

where $f(r)$ is an arbitrary function of its argument $r$. In view of Eq. (1.17) we have

$$
f(r) \mathrm{e}^{-\psi}=\frac{K(r) R^{2}}{L(x)} .
$$

Eq. (1.8) may be written as

$$
\varphi_{r}=-\frac{G_{r}}{G}-\frac{1}{x G(x)} \frac{K_{r}}{K} .
$$

If, instead of postulating a caloric equation of state and assuming that mass is conserved, we assume that an equation of state of the form

$$
p=p(w)
$$


holds, then we may define a function $\sigma$ by the equation

$$
\frac{d \sigma}{\sigma}=\frac{d w}{w+p}
$$

Eqs. (1.8) and (1.9) may be integrated to give

$$
\begin{aligned}
\mathrm{e}^{\varphi} & =\frac{g(t) \sigma}{w+p}, \\
\mathrm{e}^{\psi} & =\frac{f(r)}{R^{2} \sigma}
\end{aligned}
$$

$f$ and $g$ being arbitrary functions of their arguments. Such an equation of state will exist for isentropic flows $(K(r)$, a constant) and in that case

$$
\sigma=\varrho c^{2} \text {. }
$$

The velocity of sound $a$ in a fluid is given by the equation

$$
a^{2}=c^{2} \frac{d p}{d w}
$$

where the right hand side is evaluated for constant entropy. It follows from the above results that when a caloric equation of state holds we have

$$
\frac{a^{2}}{c^{2}}=-\frac{\frac{d G}{d x} / G}{\frac{d L}{d x} / L} .
$$

When the assumption of the existence of a caloric equation of state is replaced by the assumption that Eq. (1.20) holds, then the velocity of sound may be evaluated from Eqs. (1.24) and (1.20). This is so because in general we may write the pressure as a function of the energy density and the entropy. Eq. (1.20) states that we are assuming that for the fluid obeying this equation, the entropy does not enter into the evaluation of the pressure.

\section{Integrals of Eqs. (1.6), (1.4), and (1.13)}

If one substitutes from Eqs. (1.8) and (1.9) into Eq. (1.6) one obtains

$$
(w+p) R^{2} R_{r t}+R^{2} R_{t} p_{r}+R^{2} R_{r} w_{t}+2(w+p) R R_{r} R_{t}=0 .
$$

We may write the above equations as

$$
\left(w R^{2} R_{r}\right)_{t}+\left(p R^{2} R_{t}\right)_{r}=0 .
$$


Hence there exists a function $m(r, t)$ such that

$$
\begin{aligned}
& m_{r}=\frac{4 \pi}{c^{2}} G w R^{2} R_{r}, \\
& m_{t}=-\frac{4 \pi}{c^{2}} G p R^{2} R_{t} .
\end{aligned}
$$

Next turn to Eqs. (1.3) and (1.4), which may be written as

$$
\begin{aligned}
& {\left[R\left(1+\mathrm{e}^{-2 \varphi} \frac{R_{t}^{2}}{c^{2}}-\mathrm{e}^{-2 \psi} R_{r}^{2}\right)\right]_{r}=\frac{\kappa w}{c^{2}} R^{2} R_{r}=\frac{2 G m_{r}}{c^{2}},} \\
& {\left[R\left(1+\mathrm{e}^{-2 \varphi} \frac{R_{t}^{2}}{c^{2}}-\mathrm{e}^{-2 \psi} R_{r}^{2}\right)\right]_{t}=-\frac{\kappa p}{c^{2}} R^{2} R_{t}=\frac{2 G m_{t}}{c^{2}} .}
\end{aligned}
$$

These two equations may be immediately integrated to give

$$
\frac{2 G m}{c^{2}}=R\left(1+\mathrm{e}^{-2 \varphi} \frac{R_{t}^{2}}{c^{2}}-\mathrm{e}^{-2 \psi} R_{r}^{2}\right) .
$$

The function $m(r, t)$ satisfies Eqs. (2.1) and (2.2) and hence is determined up to an additive constant, a constant of integration.

\section{The Similarity Requirement}

We may choose our units so that

$$
4 \pi G=c=1 .
$$

This is equivalent to introducing a constant length $L_{0}$, a constant energy density $w_{0}$ and a constant mass $m_{0}$ which satisfy

$$
4 \pi G w_{0} L_{0}^{2}=c^{4}
$$

and

$$
m_{0} c^{2}=4 \pi w_{0} L_{0}^{3} \text {. }
$$

In the subsequent discussion we shall measure lengths in units of $L_{0}$ and suppress this constant. Eqs. (2.1) and (2.2) become

$$
\begin{aligned}
& m_{r}=w R^{2} R_{r}, \\
& m_{t}=-p R^{2} R_{t}
\end{aligned}
$$

respectively, and Eq. (2.5) may be written as

$$
2 m=R\left(1+\mathrm{e}^{-2 \varphi} R_{t}^{2}-\mathrm{e}^{-2 \psi} R_{r}^{2}\right) .
$$


Note that Eqs. (1.8), (1.9), and (1.11) remain unaltered. Eqs. (1.4) and (1.3), which imply Eqs. (3.6), become

$$
\begin{aligned}
\mathrm{e}^{-\varphi} u_{t} & =\mathrm{e}^{-\varphi} \Gamma \varphi_{r}-p R-\frac{m}{R^{2}}, \\
\mathrm{e}^{-\psi} \Gamma_{r} & =\mathrm{e}^{-\varphi} u \psi_{t}-w R+\frac{m}{R^{2}}
\end{aligned}
$$

respectively, where

$$
\begin{gathered}
u=\mathrm{e}^{-\varphi} R_{t}, \\
\Gamma=\mathrm{e}^{-\psi} R_{r} .
\end{gathered}
$$

The line element may now be written as

$$
d s^{2}=\mathrm{e}^{2 \varphi} d t^{2}-\mathrm{e}^{2 \psi} d r^{2}-R^{2} d \Omega^{2} .
$$

We define a spherically symmetric similarity solution of the field equations as one for which under the transformation

$$
\bar{t}=a t, \quad \bar{r}=a r, \quad \bar{\theta}=\theta, \quad \bar{\chi}=\chi
$$

where $a$ is a constant

$$
\bar{g}_{\mu \nu}(\bar{r}, \bar{t})=g_{\sigma \tau} \frac{\partial x^{\sigma}}{\partial \bar{x}^{\mu}} \frac{\partial x^{\tau}}{\partial \bar{x}^{v}}=\frac{1}{a^{2}} g_{\mu \nu}(\bar{r}, \bar{t}) .
$$

That is, such a solution gives a space-time which admits the transformation (3.11) as a conformal transformation. We shall also require that the barred coordinate system be a co-moving one.

These two requirements may be given a general and invariant formulation. We shall define a similarity solution of the field equations as one for which the resulting space-time admits the conformal Killing vector field $\xi^{\mu}$ satisfying

$$
\xi_{\mu ; \nu}+\xi_{v ; \mu}=2 g_{\mu \nu} .
$$

In case the source of the gravitational field is a perfect fluid, it is a consequence of this condition and the transformation properties of the Einstein tensor that the four-velocity vector $u^{\mu}$ is conformally invariant. That is

$$
u_{; v}^{\mu} \xi^{v}-\xi_{; \nu}^{\mu} u^{v}=-u^{\mu} .
$$

In the spherically symmetric case we may write

$$
\xi^{\mu}=\alpha \delta_{1}^{\mu}+\beta \delta_{4}^{\mu}
$$


where we have set $r=x^{1}$ and $t=x^{4}$. Eqs. (3.13) become

$$
\begin{gathered}
\alpha R_{r}+\beta R_{t}=R, \\
\alpha \psi_{r}+\beta \psi_{t}+\alpha_{r}=1, \\
\alpha \varphi_{r}+\beta \varphi_{t}+\beta_{t}=1, \\
-\mathrm{e}^{2 \psi} \alpha_{t}+\mathrm{e}^{2 \varphi} \beta_{r}=0 .
\end{gathered}
$$

Eqs. (3.14) reduce to two equations, one of these is the third one in the above set and the other is

$$
\alpha_{t}=0
$$

It then follows from the fourth of Eqs. (3.16) that

$$
\beta_{r}=0 \text {. }
$$

If we define new independent variables $\bar{r}$ and $\bar{t}$ by the equations

$$
\bar{r}_{r}=\frac{\bar{r}}{\alpha}, \quad \bar{t}_{t}=\frac{\bar{t}}{\beta}
$$

and new dependent variables

$$
\begin{aligned}
& \bar{\psi}=\psi+\log \alpha-\log \bar{r}, \\
& \bar{\varphi}=\varphi+\log \beta-\log \bar{t}
\end{aligned}
$$

the first three of Eqs. (3.16) become

$$
\begin{aligned}
& \bar{r} R_{\bar{r}}+\bar{t} R_{\bar{t}}=R \\
& \bar{r} \bar{\psi}_{r}+\bar{t} \bar{\psi}_{\bar{t}}=0 \\
& \bar{r} \bar{\varphi}_{\bar{r}}+\bar{t} \bar{\varphi}_{\bar{t}}=0 .
\end{aligned}
$$

That is, we may write

$$
\begin{aligned}
& \bar{\varphi}=\bar{\varphi}(z) \\
& \bar{\psi}=\bar{\psi}(z) \\
& \bar{R}=\bar{r} \mathscr{R}(z)
\end{aligned}
$$

where

$$
z=\bar{r} / \bar{t} .
$$

The line element may now be written as

$$
d s^{2}=\mathrm{e}^{2 \bar{\varphi}} d \bar{t}^{2}-\mathrm{e}^{2 \bar{\psi}} d \bar{r}^{2}-\bar{r}^{2} \mathscr{R}^{2} d \Omega^{2}
$$

and $\bar{r}$ and $\bar{t}$ are comoving coordinates. Hence Eqs. (3.4) to (3.10) hold in these coordinates. We shall restrict ourselves to this comoving coordinate system in the sequel and not use the bar to denote it. 
It follows from the above equations and Eqs. (3.6), (3.5) and (3.4) that

$$
\begin{aligned}
m & =r \mathscr{M}(z) \\
p & =\frac{1}{r^{2}} \mathscr{P}(z) \\
w & =\frac{1}{r^{2}} \mathscr{W}(z) .
\end{aligned}
$$

Eqs. (3.9) and (3.10) then imply that

$$
\begin{gathered}
u=\mathscr{U}(z) \\
\Gamma=\gamma(z) .
\end{gathered}
$$

It follows from the above expressions for $p$ and $w$ that $x$ defined by

$$
w+p=p x G(x)
$$

is a function of $z$ alone,

$$
x=x(z),
$$

and hence so is the temperature. Then we have

$$
\varrho=\frac{1}{r^{2}} \Omega(z)
$$

as follows from the definition of $\varrho$ in terms of $p$ and $x$. We observe that an equation of state of the form $p=p(w)$ is inconsistent with the last two of Eqs. (3.22) unless

where $\alpha$ is a constant.

$$
w=\alpha p
$$

In this case

$$
p+w=(\alpha+1) p
$$

and we can define the function $G(x)$ by the relation

$$
x G(x)=\alpha+1 .
$$

Thus for a similarity solution only equations of state of the form given by Eq. (3.24) are possible and these may be characterized by the caloric equation of state with the function $G(x)$ given by Eq. (3.25).

We note that for a similarity solution the quantities

$$
\begin{aligned}
& M=\frac{m}{R}=\frac{\mathscr{M}}{\mathscr{R}} \\
& P=p R^{2}=\mathscr{P} \mathscr{R}^{2} \\
& W=w R^{2}=\mathscr{W} \mathscr{R}^{2} \\
& N=\varrho R^{2}=\Omega \mathscr{R}^{2}
\end{aligned}
$$

all functions of $z$ alone, and

$$
N x=P .
$$




\section{The Ordinary Differential Equations}

In this section we shall show that the Einstein field equations reduce to ordinary differential equations when the space-time is one determined by a spherically symmetric similarity solution, that is, admits a vector $\xi^{\mu}$ satisfying Eqs. (3.13). We shall first rewrite the full equations in terms of the variables $M, P, W$ defined by equations (3.26) and $\mathscr{R}=R / r$ but shall consider these variables as functions of $r$ and $z=r / t$ rather than functions of $r$ and $t$.

We note that for any function $f(r, t)$ we may write

$$
\begin{aligned}
& f_{r}=\frac{\partial f}{\partial r}+\frac{\partial f}{\partial z} \frac{1}{t}, \\
& f_{t}=-\frac{1}{t} z \frac{\partial f}{\partial z},
\end{aligned}
$$

where the partial derivatives on the right hand side of these equations are taken keeping one of the variables $r$ or $z$ fixed. Hence we have

$$
\begin{aligned}
r f_{r} & =\dot{*}+\dot{f} \\
t f_{t} & =-\dot{f}
\end{aligned}
$$

where

$$
\begin{aligned}
& \stackrel{*}{f}=r \frac{\partial f}{\partial r} \\
& \dot{f}=z \frac{\partial f}{\partial z} .
\end{aligned}
$$

In particular since

$$
\begin{aligned}
R & =r \mathscr{R}(r, z) \\
R_{r} & =\mathscr{R}+\dot{\mathscr{R}}+\mathscr{R} \\
R_{t} & =-z \dot{\mathscr{R}} .
\end{aligned}
$$

Hence Eq. (3.6) may be written as

$$
2 M=1+e^{-2 \varphi} z^{2} \dot{\mathscr{R}}^{2}-e^{-2 \psi}(\mathscr{R}+\mathscr{\mathscr { R }}+\dot{\mathscr{R}})^{2}
$$

Eqs. (3.4) and (3.5) are equivalent to the equations

$$
\mathscr{R} \dot{M}+(P+M) \dot{\mathscr{R}}=0
$$

and

$$
M-W-(W+P) \frac{\dot{\mathscr{R}}}{\mathscr{R}}=(W-M) \frac{\stackrel{*}{\mathscr{R}}}{\mathscr{R}}-\stackrel{*}{M} .
$$


The integrability condition of these last two equations for determining $M(r, z)$ is equivalent to Eq. (1.6). It may be written as

$$
\ddot{\mathscr{R}}+\dot{\mathscr{R}}-(\mathscr{R}+\dot{\mathscr{R}}+\stackrel{*}{\mathscr{R}}) \dot{\psi}-\dot{\mathscr{R}} \dot{\varphi}=\dot{\mathscr{R}} \ddot{\varphi}-\ddot{\mathscr{R}} .
$$

We shall also make use of the equation obtained by subtracting equation (1.4) from (1.3). It may be shown to be

$$
\begin{aligned}
(\mathscr{R}+\stackrel{*}{\mathscr{R}}) & \dot{\varphi}-z^{2} e^{2 \psi-2 \varphi}(\mathscr{R}+\stackrel{*}{\mathscr{R}}) \dot{\psi}-e^{2 \psi} \frac{(W+P)}{\mathscr{R}} \\
= & \stackrel{*}{\mathscr{R}}+\stackrel{*}{\mathscr{R}}+\stackrel{*}{\mathscr{R}}-\stackrel{*}{\psi}(\mathscr{R}+\stackrel{*}{\mathscr{R}}+\dot{\mathscr{R}})-(\mathscr{R}+\stackrel{*}{\mathscr{R}}) \stackrel{*}{\varphi}-z^{2} e^{2 \psi-2 \varphi}(\stackrel{*}{\mathscr{R}}-\stackrel{*}{\varphi} \dot{\mathscr{R}}) .
\end{aligned}
$$

Eqs. (1.8) and (1.9) may be written as

$$
\dot{\varphi}+\frac{\dot{P}}{P+W}-\frac{2 P(\mathscr{R}+\dot{\mathscr{R}})}{(P+W) \mathscr{R}}=\frac{1}{P+W}\left(2 P \frac{\mathscr{R}}{\mathscr{R}}-\stackrel{*}{P}\right)-\stackrel{*}{\varphi}
$$

and

$$
\dot{\psi}+\frac{\dot{W}}{P+W}+\frac{2 P}{P+W} \frac{\dot{\mathscr{R}}}{\mathscr{R}}=0 .
$$

It is evident that if $\varphi, \psi, P, W, M$ and $\mathscr{R}$ are functions of $z$ alone, as they must be for a similarity solution, Eqs. (4.4) to (4.10) reduce to the following algebraic or ordinary differential equations:

$$
\begin{gathered}
2 M=1+e^{-2 \varphi} z^{2} \dot{\mathscr{R}}^{2}-e^{-2 \psi}(\mathscr{R}+\dot{\mathscr{R}})^{2}, \\
\mathscr{R} \dot{M}+(P+M) \dot{\mathscr{R}}=0, \\
M=W+(W+P) \frac{\dot{\mathscr{R}}}{\mathscr{R}}, \\
\ddot{\mathscr{R}}+\dot{\mathscr{R}}-(\mathscr{R}+\dot{\mathscr{R}}) \dot{\psi}-\dot{\mathscr{R}} \dot{\varphi}=0, \\
e^{-2 \psi} \dot{\varphi}-z^{2} e^{-2 \psi} \dot{\psi}=\frac{W+P}{\mathscr{R}^{2}}, \\
\dot{\varphi}=-\frac{\dot{P}}{W+P}+\frac{2 P}{W+P}\left(\frac{\mathscr{R}+\dot{\mathscr{R}}}{\mathscr{R}}\right)
\end{gathered}
$$

and Eq. (4.10).

In case the material is characterized by a function $G(x)$, that is, if,

$$
W+P=P x G(x)
$$

then Eq. (1.17) holds. That is,

$$
P=\frac{K(r) r^{2} \mathscr{R}^{2}}{x L(x)} .
$$


Since $\mathscr{R}, P$, and $x$ must be functions of $z$ alone, we must have

$$
r^{2} K(r)=K_{0}
$$

for a similarity solution and hence

$$
\begin{gathered}
P=\frac{K_{0} \mathscr{R}^{2}}{x L(x)}, \\
W+P=K_{0} \frac{G(x)}{L(x)} \mathscr{R}^{2} .
\end{gathered}
$$

Eq. (1.18) with $f(r)=f_{0}$, a constant is then the integral of Eq. (4.10). That is

$$
e^{-\psi}=\frac{B \mathscr{R}^{2}}{L(x)} .
$$

$B$ a constant, and Eq. (4.16) becomes

$$
\dot{\varphi}=-\frac{1}{G} \frac{d G}{d x} \dot{x}+\frac{2}{x G(x)} .
$$

Eqs. (4.14) and (4.15) may now be written as differential equations for $\mathscr{R}$ and $x$. Thus the former equation may be written as

$\ddot{\mathscr{R}}+\dot{\mathscr{R}}-\frac{\dot{x}}{L} \frac{d L}{d x}\left[\mathscr{R}+\left(1+a^{2}\right) \dot{\mathscr{R}}\right]+\frac{2 \dot{\mathscr{R}}}{\mathscr{R}}\left[\mathscr{R}+\dot{\mathscr{R}}-\frac{\mathscr{R}}{x G(x)}\right]=0$,

where $a^{2}$ is the velocity of sound given by Eq. (1.25), and is a function of $x$. Eq. (4.15) becomes

$$
\frac{2}{x G(x)}+2 z^{2} e^{2 \psi-2 \varphi} \frac{\dot{\mathscr{R}}}{\mathscr{R}}-\frac{\dot{x}}{L} \frac{d L}{d x}\left[z^{2} e^{2 \psi-2 \varphi}-a^{2}\right]-K_{0} \frac{e^{2 \psi} G(x)}{L(x)}=0 .
$$

The system of Eqs. (4.20) through (4.23) together with appropriate initial conditions, that is appropriate constants of integration, determine a similarity solution.

Although the system of Eqs. (4.20) through (4.23) has six constants of integration, including the constants $B$ and $K_{0}$, they are not all independent in a similarity solution since Eqs. (4.11) and (4.13) imply that we must have

$$
F(z) \equiv 1+e^{-2 \varphi} z^{2} \dot{\mathscr{R}}^{2}-e^{-2 \psi}(\mathscr{R}+\dot{\mathscr{R}})^{2}-2\left(W+(W+P) \frac{\dot{\mathscr{R}}}{\mathscr{R}}\right)=0
$$

satisfied for all values of $z$. In particular this equation must hold for the initial value of $z$ used in the integration of the system consisting of Eqs. (4.20) through (4.23). This implies that the similarity solution 
depends on five parameters, namely the constant $K_{0}$ which determines the entropy function in the similarity region and the four independent constants of integration mentioned above. We shall show below that two of the latter constants are inessential ones in the sense that they may be given arbitrary values by still another linear coordinate transformation involving the variable $r$ and $t$ (or $z$ ).

Before discussing this point we observe that if Eq. (4.24) is satisfied for one value of $z$ then Eqs. (4.10) through (4.16) imply that it is satisfied for all values of $z$. For it may be verified from the latter equations that

$$
\dot{F}(z)=0 \text {. }
$$

We close this section with a discussion of the number of essential parameters in a similarity solution. As we have seen for such a solution the line element is of the form

$$
d s^{2}=e^{2 \varphi} d t^{2}-e^{2 \psi} d r^{2}-r^{2} \mathscr{R}^{2} d \Omega^{2},
$$

where $\varphi, \psi$, and $\mathscr{R}$ are functions of $z=r / t$. Under the transformation

$$
t=\beta \hat{t}, \quad r=\alpha \hat{r}
$$

which we shall call a'scale transformation, we may write

$$
d s^{2}=e^{2 \hat{\varphi}} d \hat{t}^{2}-e^{2 \hat{\psi}} d \hat{r}^{2}-\hat{r}^{2} \hat{R}^{2} d \Omega^{2}
$$

where $\hat{\varphi}, \hat{\psi}$ and $\hat{\mathscr{R}}$ are functions of the variable

$$
\hat{z}=\frac{\beta}{\alpha} z
$$

defined by the equations

$$
\begin{aligned}
& e^{\hat{\varphi}(\hat{z})}=\beta e^{\varphi\left(\frac{\alpha}{\beta} \hat{z}\right)}=\beta e^{\varphi(z)} \\
& e^{\hat{\psi}(\hat{z})}=\alpha e^{\psi\left(\frac{\alpha}{\beta} \hat{z}\right)}=\alpha e^{\psi(z)} \\
& \hat{\mathscr{R}}(\hat{z})=\alpha \mathscr{R}\left(\frac{\alpha}{\beta} \hat{z}\right)=\alpha \mathscr{R}(z) .
\end{aligned}
$$

Quantities such as $\varphi, \psi$ and $\mathscr{R}$ whose transformation law under a scale transformation involves the coefficients of this transformation, explicitly will be said to be scale covariants. The constants of integration which enter into the expression of two scale covariants may be transformed to any desired values by a scale transformation. We shall therefore call such constants inessential parameters in the similarity solution. We 
next show that the differential equations describing a similarity solution may be decomposed into two sets, one involving scale covariants and one involving scale invariants. The latter quantities are such that their functional form and value are unaltered by a scale transformation.

Examples of scale invariants are

and

$$
V=e^{\psi-\varphi} z
$$

$$
H=V e^{-\psi} \mathscr{R}
$$

It may readily be verified that as a consequence of equations (4.26) and (4.27)

$$
\hat{V} \equiv e^{\hat{\psi}-\hat{\varphi}} \hat{z}=e^{\psi-\varphi} z=V
$$

and that

$$
\hat{H} \equiv \hat{V} e^{-\hat{\psi}} \hat{\mathscr{R}}=V e^{-\psi} \mathscr{R}=H .
$$

If $G(z)$ is any scalar function, then

$$
\dot{G}=z \frac{d G}{d z}=\hat{z} \frac{d \hat{G}}{d \hat{z}}
$$

and is a scale invariant. Although $\mathscr{R}(z)$ is not a scale invariant, the quantities $\dot{\mathscr{R}} / \mathscr{R}, \dot{\varphi}$ and $\dot{\psi}$ are.

The hypersurface $z=$ constant has the equation

$$
r-z t=0,
$$

and the normal vector oriented into the future with components

$$
n_{\mu}=z \delta_{\mu}^{4}-\delta_{\mu}^{1} .
$$

It may be verified that

$$
V=\frac{u^{\mu} n_{\mu}}{\sqrt{\left(n_{\mu} u^{\mu}\right)^{2}-n_{\mu} n^{\mu}}}
$$

and represents the normal velocity relative to the fluid of the moving sphere of radius $z t$. Similarly, the scale invariant

$$
\tanh \omega=e^{\psi-\varphi} \frac{R_{t}}{R_{r}}=-\frac{V \dot{\mathscr{R}}}{\mathscr{R}+\dot{\mathscr{R}}}=\frac{u^{\mu} n_{\mu}}{\sqrt{\left(n_{\mu} u^{\mu}\right)^{2}-n_{\mu} n^{\mu}}}
$$

represents the normal velocity of the fluid relative to the sphere $r=r(t)$ obtained by solving the equations

$$
R(r, t)=\text { constant } .
$$

In this case

$$
n_{\mu}=R_{t} \delta_{\mu}^{4}+R_{r} \delta_{\mu}^{1}
$$


It is a consequence of the remarks made above and Eqs. (4.11), (4.12) and (4.13) that $M, P, W, x$, and $N$ are scale invariants. Moreover it may be verified from these equations that

$$
\tanh \omega=\frac{V(W-M)}{M+P}
$$

and that

$$
H^{2}=\frac{(1-2 M)(W+P)^{2} V^{2}}{(P+M)^{2}-(W-M)^{2} V^{2}} .
$$

Eqs. (4.10) through (4.16) involve only scale invariant quantities as may readily be verified. However the constants $K_{0}$ and $B$ entering into Eqs. (4.18), (4.19), and (4.20) are scale dependent. The first of these equations may be written as

$$
N=\frac{K_{0} \mathscr{R}^{2}}{L(x)}=\frac{K_{0}}{B} e^{-\psi} .
$$

But from Eq. (4.20) we have

$$
e^{-\psi}=\frac{B \mathscr{R}^{2}}{L(x)}=\frac{B e^{2 \psi}}{L(x)} \frac{H^{2}}{V^{2}} .
$$

Hence we may write

$$
N^{3} L(x) \frac{V^{2}}{H^{2}}=\frac{K_{0}^{3}}{B^{2}}=K_{1}
$$

a scale invariant constant.

It may be shown that as a consequence of Eqs. (4.12) to (4.18)

$$
\begin{aligned}
\dot{P} & =\sigma_{1}(P, x, M) \\
\dot{x} & =\sigma_{2}(P, x, M) \\
\dot{M} & =\sigma_{3}(P, x, M)
\end{aligned}
$$

where

$$
\begin{aligned}
& \sigma_{1}=\left\{\frac{-2 P}{W+P}\left[\frac{W-M}{\frac{x}{G} \frac{d G}{d x}}+\frac{P+M}{V^{2}}\right]+\left(\frac{W+P}{H}\right)^{2}\right\} \frac{a^{2} V^{2}}{V^{2}-a^{2}} \\
& \sigma_{2}=\frac{1}{x \frac{d G}{d x}}\left[\frac{\sigma_{1}}{P}+2 \frac{(W-M)}{W+P}\right] \\
& \sigma_{3}=\frac{(W-M)(M+P)}{W+P} .
\end{aligned}
$$


In these equations $W$ and $N$ may be regarded as functions of $P$ and $x$, and $V$ and $H$ are given as functions of $P, x$ and $M$ by means of Eqs. (4.31) and (4.32).

Eqs. (4.33) may be written as

$$
\begin{aligned}
& \frac{d P}{d M}=\frac{\sigma_{1}}{\sigma_{3}} \\
& \frac{d x}{d M}=\frac{\sigma_{2}}{\sigma_{3}}
\end{aligned}
$$

and their solutions and Eqs. (4.32) may be written as

$$
\begin{aligned}
P & =P\left(K_{i} ; M\right) \\
x & =x\left(K_{i} ; M\right) \quad i=1,2,3, \\
V & =V\left(K_{i} ; M\right)
\end{aligned}
$$

where $K_{1}$ is the constant appearing in Eq. (4.32) and $K_{2}$ and $K_{3}$ are two scale invariant constants of integration.

The functions $\psi, \varphi$ and $z$ are then given by

$$
\begin{aligned}
\mathrm{e}^{\psi} & =\frac{B_{0}}{N} \\
\mathrm{e}^{\varphi} & =\frac{A_{0}}{V N} \exp \left(\int \frac{d M}{\sigma_{3}}\right) \\
z & =\mathrm{e}^{\varphi-\psi} V .
\end{aligned}
$$

The constants $A_{0}$ and $B_{0}$ are scale dependent and may be assigned any convenient value by a scale transformation. They are then said to be non-essential parameters but the constants $K_{1}, K_{2}$, and $K_{3}$ are called essential ones since they are scale independent. Any two conditions which fix $A_{0}$ and $B_{0}$ are said to determine a coordinate normalization for $r$ and $t$.

Once the three constants $K_{1}, K_{2}$ and $K_{3}$ are given the similarity solution is determined. When a coordinate normalisation which determines $A_{0}$ and $B_{0}$ is given then $z$ is a known function of $M$ by means of Eqs. (4.37). Eqs. (4.36) may also be solved for the

$$
K_{i}=K_{i}(M, P, x, V),
$$

and from the preceding remarks it is seen that if the quantities $M, P, x$ and $V$ are given on a hypersurface $z=$ constant then this hypersurface and the similarity solution to which it belongs are determined. 
One observer with the world line

$$
\begin{aligned}
& r=z_{0} t, \\
& \theta=\theta_{0}, \\
& \chi=\chi_{0}
\end{aligned}
$$

with $z_{0}, \theta_{0}, \chi_{0}$ constants a $z$-observers is always in the hypersurface $z=z_{0}$. When $z_{0}>0$, the observer moves outward through the fluid toward higher values of $r$ and when $z_{0}<0$ be moves inward. When $z_{0}=0$ his world line coincides with that of the fluid element at $r=0$.

The observer with the world-line

$$
\begin{aligned}
R(r, t) & =R_{0}, \\
\theta & =\theta_{0}, \\
\chi & =\chi_{0}
\end{aligned}
$$

with $R_{0}, \theta_{0}, \chi_{0}$ constants a $R$-observers has his velocity relative to the fluid determined by $\tanh \omega$. It is evident from Eq. (4.30) that when $V>0$ and $W>M$ then tanh $\omega>0$. That is, even though the material is falling behind the $z$-observer, when $t>0$, it can be moving outward relative to the $R$-observer.

\section{Initial Data for the Field Equations}

If a similarity solution is to describe the region of space-time representing the events behind a moving shock wave, the motion of that wave must be given by the hypersurface

$$
z=z_{1}
$$

with $z_{1}$ a constant and the region in which the similarity solution holds must be given by

$$
z_{0} \leqq z \leqq z_{1}
$$

with $z_{0}$ another constant. The values of the metric tensor and other field variables on this hypersurface, together with the jump conditions, which will be discussed below, will provide information concerning the quantities that enter into the full Einstein field equations, Eqs. (4.4) through (4.10). It is therefore of interest to examine the nature of the data needed to ensure the existence and uniqueness of the solution of these equations. The usual discussion of these questions does not apply since the hypersurface involved is a time-like one. 
We first treat the case in which the fluid is characterized by the function $G(x)$. In that case Eqs. (4.9) and (4.10) become

$$
\dot{\varphi}+\frac{d G}{d x} \frac{\dot{x}}{G}=-\left(\stackrel{*}{\varphi}+\frac{d G}{d x} \frac{\stackrel{*}{x}}{G}\right)-\frac{1}{x G} \frac{\stackrel{*}{K}}{K}
$$

and

$$
\dot{\psi}=\frac{1}{L} \frac{d L}{d x} \dot{x}-\frac{2 \dot{\mathscr{R}}}{\mathscr{R}}
$$

respectively. Eq. (4.7) may be written as

$$
\begin{aligned}
& \ddot{\mathscr{R}}+\dot{\mathscr{R}}-\frac{1}{L} \frac{d L}{d x} \dot{x}\left[\mathscr{R}+\mathscr{\mathscr { R }}+\left(1+a^{2}\right) \dot{\mathscr{R}}\right] \\
& +\frac{\dot{\mathscr{R}}}{\mathscr{R}}\left[2(\mathscr{R}+\dot{\mathscr{R}}+\stackrel{*}{\mathscr{R}})+\mathscr{R}\left(\frac{1}{G} \frac{d G}{d x} \stackrel{*}{x}+\frac{\stackrel{*}{K}}{x G(x) K}\right)\right]=\dot{\mathscr{R}} \stackrel{*}{\psi}-\dot{\mathscr{R}}^{*}
\end{aligned}
$$

and Eq. (4.8) as,

$$
\begin{aligned}
(\mathscr{R} & +\mathscr{\mathscr { R }}) \frac{1}{L} \frac{d L}{d x} \dot{x}\left(V^{2}-a^{2}\right)=-\mathrm{e}^{2 \psi} \frac{r^{2} K(r) \mathscr{R} G(x)}{L(x)}-\stackrel{* *}{\mathscr{R}}-\ddot{\mathscr{R}}-\mathscr{*} \\
& +(\mathscr{R}+\stackrel{*}{\mathscr{R}})\left(2 V^{2} \frac{\dot{\mathscr{R}}}{\mathscr{R}}-\frac{1}{G} \frac{d G}{d x} \stackrel{*}{x}-\frac{\stackrel{*}{K}}{x G(x) K}+\stackrel{*}{\varphi}\right)+\stackrel{*}{\psi}(\mathscr{R}+\stackrel{*}{\mathscr{R}}+\stackrel{\dot{\mathscr{R}}}{x}) \\
& +V^{2}(\stackrel{*}{\mathscr{R}}-\stackrel{*}{\varphi} \dot{\mathscr{R}}),
\end{aligned}
$$

when $V$ is defined by Eq. (4.28).

If we define

$$
\begin{aligned}
& Y^{1}=\mathscr{R}, \\
& Y^{2}=\dot{\mathscr{R}}, \\
& Y^{3}=\mathscr{R}, \\
& Y^{4}=x, \\
& Y^{5}=\varphi, \\
& Y^{6}=\psi
\end{aligned}
$$

then we have

$$
\begin{aligned}
& \dot{Y}^{1}=Y^{2}, \\
& \dot{Y}^{3}=\dot{Y}^{2},
\end{aligned}
$$

and Eqs. (5.1) through (5.6) may be written as

$$
\dot{Y}^{A}=F^{A}\left(Y^{B} ; \stackrel{*}{Y}^{B} ; r ; z\right) .
$$

The Cauchy-Kowaleski theorem may then be applied to this system of equations. From it we may deduce that if the initial hypersurface 
$z=z_{1}$ is such that

$$
(\mathscr{R}+\stackrel{*}{\mathscr{R}})\left(V^{2}-a^{2}\right) \neq 0
$$

then the equations have a unique and analytic solution in the neighbourhood of this hypersurface, determined by the values of $Y^{A}$ and $\stackrel{Y}{ }^{A}$ on the hypersurface. These quantities constitute the initial data of the problem. If they are restricted so that Eq. (4.6) is satisfied we will have a solution of the Einstein field equations. The latter equation may be written as

$$
G\left(Y^{A} ; \stackrel{*}{Y}^{A} ; r ; z\right)=0
$$

since $M, W$ and $P$ may be expressed in terms of the variables $Y^{A}$ and $\stackrel{*}{Y}^{A}$ Eq. (4.5) follows as a consequence of Eqs. (4.6), (4.7), and (4.8) and the definition of $M$.

The hypersurfaces for which

$$
V^{2}-a^{2}=0
$$

are the hydrodynamical characteristic surfaces. They describe the moving wavefronts of sound waves.

The hypersurfaces for which

$$
\mathscr{R}+\stackrel{*}{\mathscr{R}}=0
$$

are such that

$$
1-2 M=\left(1-V^{2}\right) \mathrm{e}^{-2 \psi} \dot{\mathscr{R}}^{2} .
$$

It is evident that if the condition (5.8) holds on and in the neighbourhood of an initial hypersurface $z=z_{1}$, and if on this hypersurface the $\stackrel{*}{Y}^{A}$ vanish and $K r^{2}=k_{0}$ a constant, then a solution of the field equations is provided by a similarity solution. Because the solution is unique we may conclude that the only solution of the Einstein field equations which takes on constant values and for which $K r^{2}=K_{0}$, a constant on a hypersurface $z=z_{1}$ is a similarity solution. For such a solution Eqs. (5.7) are of the form

$$
\dot{Y}^{A}=F^{A}\left(Y^{B} ; z\right)
$$

Where an equation of state of the form

$$
p=p(w)
$$

exists, we may write Eqs. (1.22) and (1.23) as

$$
\mathrm{e}^{\varphi}=\frac{g(t) \sigma}{w+p}
$$


and

$$
\mathrm{e}^{\psi}=\frac{f_{1}(r)}{\mathscr{R}^{2} \sigma}
$$

Then by means of Eq. (1.21)

$$
\begin{aligned}
& \dot{\varphi}=-\frac{\dot{g}}{g}-a^{2} \frac{\dot{\sigma}}{\sigma} \\
& \stackrel{*}{\varphi}=\frac{\dot{g}}{g}-a^{2} \frac{\stackrel{\sigma}{\sigma}}{\sigma} \\
& \dot{\psi}=-\frac{\dot{\sigma}}{\sigma}-\frac{2 \mathscr{R}}{\mathscr{R}} \\
& \ddot{\psi}=-\frac{\dot{\sigma}}{\sigma}-\frac{2 \mathscr{R}}{\mathscr{R}}-\frac{\dot{f}_{1}}{f_{1}}
\end{aligned}
$$

where

$$
a^{2}=\frac{d p}{d w}=a^{2}(\sigma)
$$

It may be verified that if Eqs. (5.13) are substituted into Eqs. (4.7) and (4.8), these equations and Eqs. (5.5) and (5.6) may be written in the form

$$
\dot{Y}^{A}=F^{A}\left(Y^{B} ; \stackrel{*}{Y}^{B} ; r, z\right) \quad A, B=1, \ldots, 4
$$

where now

$$
\begin{aligned}
Y^{1} & =\mathscr{R}, \\
Y^{2} & =\dot{\mathscr{R}}, \\
Y^{3} & =\mathscr{R}, \\
Y^{4} & =\sigma .
\end{aligned}
$$

We may again apply the Cauchy-Kowaleski theorem and obtain an existence and uniqueness theorem when the condition (5.8) obtains. A solution of the Einstein field equations is given when the initial data satisfies Eq. (4.6) which is again an equation of the form of Eq. (5.9).

The initial data may be provided by data for which the $\stackrel{I}{Y}^{A}$ vanish on a hypersurface $z=z_{1}$ and the functions $\varphi$ and $\psi$ are constant. The latter requirement serves to determine the functions $f_{1}(r)$ and $g(t)$ in terms of the initial values of $\mathscr{R}$ and $\sigma(r, z)$. If $p$ is not proportional to $w$, the solution determined by such data is not a similarity solution for we have seen that such equations of state do not allow a similarity solution to hold. 


\section{The Conditions across a Spherical Shock}

In this section we shall discuss the conditions which relate the metric tensor and the hydrodynamic variables on the two sides of a singular hypersurface which represents the motion of a shock wave in the spacetime with the line element given by Eq. (1.1). This hypersurface will be assumed to separate space-time into two regions. Later we shall take one of these regions to be that described by the similarity solution discussed above and the other to be described by a known solution of the Einstein field equations.

In general comoving coordinates, the equation specifying the spherical shock is given by

$$
r=\Lambda(t)
$$

If the shock is a hypersurface $z=z_{1}$ in the special comoving coordinates we introduced in the discussion of the similarity solution, we shall have

$$
\Lambda(t)=z_{1} t
$$

where $z_{1}$ is a constant.

The interpretation of various quantities that occur in the discussion given below is attained from the use of another coordinate system in the spherical space-time, namely Schwarzschild coordinates in which the line-element given by Eq. (1.1) has the form

$$
d s^{2}=\mathrm{e}^{v} d T^{2}-\mathrm{e}^{\lambda} d R^{2}-R^{2} d \Omega^{2} .
$$

The $R, T$ coordinates are related to the comoving ones $r, t$ by the equations

$$
\begin{aligned}
& d R=\mathrm{e}^{-\lambda / 2}\left(\mathrm{e}^{\varphi} \sinh \omega d t+\mathrm{e}^{\psi} \cosh \omega d r\right) \\
& d T=\mathrm{e}^{-v / 2}\left(\mathrm{e}^{\varphi} \cosh \omega d t+\mathrm{e}^{\psi} \sinh \omega d r\right)
\end{aligned}
$$

where

$$
\begin{gathered}
\tanh \omega=\mathrm{e}^{\psi-\varphi} \frac{R_{t}}{R_{r}}, \\
\mathrm{e}^{-\lambda}=\mathrm{e}^{-2 \psi} R_{r}^{2}-\mathrm{e}^{-2 \varphi} R_{t}^{2}=1-\frac{2 m}{R}
\end{gathered}
$$

and $v$ is such that the right hand side of the second of Eqs. (6.4) is a perfect differential.

In the Schwarzschild coordinates we have the four-velocity vector given by

$$
U^{\mu}=u^{\sigma} \frac{\partial x^{* \mu}}{\partial x^{\sigma}}=\mathrm{e}^{-\varphi} \frac{\partial x^{* \mu}}{\partial t},
$$


that is, $U^{2}=U^{3}=0$ and

$$
\begin{aligned}
& U^{4}=\mathrm{e}^{-v / 2} \cosh \omega, \\
& U^{1}=\mathrm{e}^{-\lambda / 2} \sinh \omega .
\end{aligned}
$$

That is, $\omega$ is related to the proper velocity associated with an element of the fluid as measured in the Schwarzschild coordinate system. In this coordinate system the hypersurface described by Eq. (6.2) would be given by

$$
R=\mathscr{T}(T)
$$

and its unit normal will have the components $N_{\mu}$ where $N_{2}=N_{3}=0$ and

where

$$
N_{4}=\mathrm{e}^{v / 2} \sinh C ; \quad N_{1}=-\mathrm{e}^{\lambda / 2} \cosh C
$$

$$
\tanh C=\mathrm{e}^{\lambda / 2-v / 2} \mathscr{T}_{T} .
$$

That is, $C$ determines the proper velocity of the shock front in this coordinate system.

Then

$$
U^{\mu} N_{\mu}=\sinh (C-\omega)=u^{\mu} n_{\mu}
$$

where $n_{\mu}$ is the unit normal to the shock front in the comoving coordinate system. That is $n_{2}=n_{3}=0$ and

$$
n_{4}=\mathrm{e}^{\varphi} \sinh (C-\omega) ; \quad n_{1}=-\mathrm{e}^{\psi} \cosh (C-\omega)
$$

with

$$
\tanh (C-\omega)=\mathrm{e}^{\psi-\varphi} \Lambda_{t} .
$$

The quantity $C-\omega$ measures the velocity of the shock front relative to the flow.

We shall use the notation

$$
\begin{aligned}
& f_{-}=\lim _{\varepsilon \rightarrow 0} f(\mathscr{T}(T)-\varepsilon, T), \\
& f_{+}=\lim _{\varepsilon \rightarrow 0} f(\mathscr{T}(T)+\varepsilon, T),
\end{aligned}
$$

and

$$
[f]=f_{-}-f_{+},
$$

where $f$ is any function of $R$ and $T$. Similarly, in the comoving coordinate system we shall define corresponding quantities, for example

$$
f_{-}=\lim _{\varepsilon \rightarrow 0} f(\Lambda(t)-\varepsilon, t) .
$$


Thus $f_{-}$and $f_{+}$are the values of the function $f$ on the two sides of the shock and $[f]$ denotes the jump in $f$ across the shock. The quantities $f_{+}$ and $f_{-}$depend on the point of the shock at which they are evaluated and will in general vary as this point is changed. They may be considered as functions of $R$ (or $r$ ) and $T$ (or $t$ ).

It may be shown that it is no restriction to assume that

$$
C_{+}=C_{-} \text {. }
$$

It then follows from Eq. (6.7) and the requirement that the induced metric on the hypersurface $R=\mathscr{T}(T)$ be continuous that $v(R, T)$ and $\lambda(R, T)$ be continuous across the shock. Thus $m(R, T)$ defined by Eq. (6.6) is continuous across the shock. In the comoving coordinate system the condition that the induced metric be continuous implies that

$$
\begin{aligned}
R_{+} & =R_{-}, \\
\left(e^{2 \varphi}-e^{2 \psi} \Lambda_{t}^{2}\right)_{+} & =\left(e^{2 \varphi}-e^{2 \psi} \Lambda_{t}^{2}\right)_{-} .
\end{aligned}
$$

Eqs. (6.9) and (6.10) then imply that

$$
e^{-\varphi_{+}} \cosh \left(C-\omega_{+}\right)=e^{-\varphi_{-}} \cosh \left(C-\omega_{-}\right)
$$

and

$$
e^{-\psi+} \sinh \left(C-\omega_{+}\right)=e^{-\varphi-} \sinh \left(C-\omega_{-}\right) .
$$

That is, in the comoving coordinate system the metric is discontinuous since $\varphi$ and $\psi$ are discontinuous, but the discontinuities in these qualities are governed by Eqs. (6.12) and (6.13) and as we shall see they may also be expressed in terms of the discontinuity of the hydrodynamic variables.

We have seen, in the discussion of the conditions that obtain across the shock in the Schwarzschild coordinate system, that the function $m$ is continuous. It is of interest that this fact may be derived from the continuity of $R(r, t)$ and Eqs. (6.5), (6.9), (6.12), and (6.13). We have by differentiating Eqs. (6.11) that

$$
R_{+r}\left(\Lambda_{t}+\frac{R_{+t}}{R_{+r}}\right)=R_{-r}\left(\Lambda_{t}+\frac{R_{-t}}{R_{-r}}\right) .
$$

On using Eqs. (6.5) and (6.9) we may write this as

$$
\left[R_{r} e^{\varphi-\psi}(\tanh (C-\omega)+\tanh \omega)\right]=0 .
$$

It then follows from Eqs. (6.12) that

$$
\left[R_{r}^{2} e^{-2 \psi}-R_{t}^{2} e^{-2 \varphi}\right]=0 .
$$

In view of the definition of $m$ and the continuity of $R$ it follows that $m$ is continuous. 
The jump in the hydrodynamic variables is described by the RankineHugoniot equations which may be written as

$$
\begin{aligned}
{\left[\varrho U^{\mu} N_{\mu}\right] } & =0, \\
{\left[\left((w+p) U^{\mu} U^{v}-p g^{\mu \nu}\right) N_{v}\right] } & =0
\end{aligned}
$$

in the Schwarzschild coordinate system where $U^{\mu}$ and $N_{\mu}$ are given as above. If we define

$$
U=U^{\mu} N_{\mu}=u^{\mu} n_{\mu}=\sinh (C-\omega)
$$

we may write these equations as

$$
\begin{aligned}
\varrho_{+} U_{+} & =\varrho_{-} U_{-} \\
U_{+}^{2}\left(w_{+}+p_{+}\right)+p_{+} & =U_{-}^{2}\left(w_{-}+p_{-}\right)+p_{-} \\
U_{+}^{2}\left(w_{+}+p_{+}\right)^{2}-U_{-}^{2}\left(w_{-}+p_{-}\right)^{2} & =\left(p_{+}-p_{-}\right)\left(U_{+}^{2}\left(w_{+}+p_{+}\right)+U_{-}^{2}\left(w_{-}+p_{-}\right)\right) .
\end{aligned}
$$

Eqs. (6.14) may be written as

$$
\begin{aligned}
U_{-}^{2} & =\frac{\left(p_{-}-p_{+}\right)\left(w_{+}+p_{-}\right)}{\left(w_{-}+p_{-}\right)\left(w_{-}-w_{+}+p_{+}-p_{-}\right)} \\
U_{+}^{2} & =\frac{\left(p_{-}-p_{+}\right)\left(w_{-}+p_{+}\right)}{\left(w_{+}+p_{+}\right)\left(w_{-}-w_{+}+p_{+}-p_{-}\right)} \\
\frac{\varrho_{-}^{2}}{\varrho_{+}^{2}}=\frac{U_{+}^{2}}{U_{-}^{2}} & =\frac{\left(w_{-}+p_{+}\right)\left(w_{-}+p_{-}\right)}{\left(w_{+}+p_{-}\right)\left(w_{+}+p_{+}\right)} .
\end{aligned}
$$

It then follows that

satisfies

$$
V^{2}=\frac{U^{2}}{1+U^{2}}=\tanh ^{2}(C-\omega)=e^{2 \psi-2 \varphi} \Lambda_{t}^{2}
$$

$$
\begin{aligned}
& V_{+}^{2}=\frac{\left(p_{-}-p_{+}\right)\left(w_{-}+p_{+}\right)}{\left(w_{-}-w_{+}\right)\left(w_{+}+p_{-}\right)}, \\
& V_{-}^{2}=\frac{\left(p_{-}-p_{+}\right)\left(w_{+}+p_{-}\right)}{\left(w_{-}-w_{+}\right)\left(w_{-}+p_{+}\right)} .
\end{aligned}
$$

Hence

$$
\tanh \left(\omega_{-}-\omega_{+}\right)=\frac{V_{+}-V_{-}}{1-V_{-} V_{+}}=\sqrt{\frac{\left(p_{-}-p_{+}\right)\left(w_{-}-w_{+}\right)}{\left(w_{+}+p_{-}\right)\left(w_{-}+p_{+}\right)}}=A
$$

and

$$
\tanh \omega_{-}=\frac{A+\tanh \omega_{+}}{1+A \tanh \omega_{+}} .
$$


Eqs. (6.12) and (6.13) may be written as

and

$$
e^{\varphi_{-} \varphi_{+}}=\frac{\left(w_{+}+p_{+}\right) \varrho_{-}}{\left(w_{-}+p_{-}\right) \varrho_{+}}=\sqrt{\frac{\left(w_{-}+p_{+}\right)\left(w_{+}+p_{+}\right)}{\left(w_{+}+p_{-}\right)\left(w_{-}+p_{-}\right)}}
$$

$$
e^{\dot{\psi}_{-}-\psi_{+}}=\frac{\varrho_{+}}{\varrho_{-}}=\sqrt{\frac{\left(w_{+}+p_{-}\right)\left(w_{+}+p_{+}\right)}{\left(w_{-}+p_{+}\right)\left(w_{-}+p_{-}\right)}}
$$

respectively.

The continuity of the function $m(r, t)$ across the shock implies that

$$
[m(\Lambda(t), t)]=0
$$

for all values of $t, \theta, \chi$ labelling a point on the shock hypersurface. That is we must have

$$
\left[m_{r} \Lambda_{t}+m_{t}\right]=0
$$

or in view of the field equations and the continuity of $R(r, t)$

$$
\left[w R_{r} \Lambda_{t}-p R_{t}\right]=0 .
$$

It may be verified that this equation is satisfied as a consequence of the equations given above.

It follows from the continuity of $m$ and $R$ across the shock that Eqs. (6.16), (6.17), and (6.21) may be written as

$$
\begin{aligned}
V_{-}^{2} & =\frac{\left(P_{-}-P_{+}\right)\left(W_{+}+P_{-}\right)}{\left(W_{-}-W_{+}\right)\left(W_{-}+P_{+}\right)}, \\
V_{+}^{2} & =\frac{\left(P_{-}-P_{+}\right)\left(W_{-}+P_{+}\right)}{\left(W_{-} W_{+}\right)\left(W_{+}+P_{-}\right)}, \\
\frac{N_{+}^{2}}{N_{-}^{2}} & =\frac{\left(W_{+}+P_{+}\right)\left(W_{+}+P_{-}\right)}{\left(W_{-}+P_{-}\right)\left(W_{-}+P_{+}\right)}
\end{aligned}
$$

respectively, and we also have

$$
M_{-}=M_{+} .
$$

If the shock is a hypersurface $z=z_{1}, a$ constant, which separates two similarity solutions each described by a function $G(x)$ where $G_{+}(x)$ may differ from $G_{-}(x)$, the above equations relate the scale invariants $P, M, x$ and $V$ on both sides of the shock. Since the values of these quantities on a hypersurface $z=$ constant determine the hypersurface and a similarity solution we see that a similarity solution on one side of a shock, and the position of the shock (the value of $z_{1}$ ) determine the similarity solution on the other side of the shock consistent with this value of $z_{1}$. The constants $K_{i}$ appearing in Eqs. (4.36) for the similarity 
solution behind the shock may then be determined in terms of those appearing in the similarity solution ahead of the shock by using Eqs. (6.23) to (6.26).

Eqs. (6.20) and (6.21), as they stand, relate $\varphi_{-}$to $\varphi_{+}$and $\psi_{-}$to $\psi_{+}$ across the shock when the same coordinates $r$ and $t$ are used on both sides. Suppose now that the coordinates on one side are scaled according to (4.25) but those on the other are left unchanged. We would then have

$$
t_{-}=t_{+}=\beta \hat{t}_{+}, \quad r_{-}=r_{+}=\alpha \hat{r}_{+} .
$$

In view of (4.27) it follows that

$$
\frac{t_{-}}{\hat{t}_{+}} e^{\varphi_{-}-\hat{\varphi}_{+}}=e^{\varphi_{-}-\varphi_{+}} ; \frac{r_{-}}{\hat{r}_{+}} e^{\psi_{--} \hat{\psi}_{+}}=e^{\psi_{-}-\psi_{+}} .
$$

Therefore when $r_{-} \neq r_{+}$and $t_{-} \neq t_{+}$because different scales are used on both sides of the shock, Eqs. (6.20) and (6.21) may be written as

$$
\frac{t_{-}}{t_{+}} e^{\varphi_{--} \varphi_{+}}=\frac{G_{+}\left(x_{+}\right)}{G_{-}\left(x_{-}\right)}=\sqrt{\frac{\left(W_{-}+P_{+}\right)\left(W_{+}+P_{+}\right)}{\left(W_{+}+P_{-}\right)\left(W_{-}+P_{+}\right)}}
$$

and

$$
\frac{r_{-}}{r_{+}} e^{\psi--\psi+}=\frac{N_{+}}{N_{-}} .
$$

Thus if $A_{0_{-}}, B_{0_{-}}, A_{0+}$ and $B_{0+}$ are chosen conveniently on each side of the shock, Eqs. (4.37) determine $z, \psi$ and $\varphi$ on both sides and (6.27) and (6.28) relate $r_{-}$to $r_{+}$and $t_{-}$to $t_{+}$.

\section{Solutions Compatible with Similarity Solutions}

The jump conditions across a shock, namely the generalised RankineHugoniot equations given by Eqs. (6.15) through (6.19) and the conditions on the metric tensor given by Eqs. (6.20) and (6.21) together with the continuity of the functions $m(r, t)$ and $R(r, t)$ may be regarded as either determining the variables behind the shock in terms of those ahead of it or vice versa. If a solution ahead of the shock is such that the variables so determined are the values that these variables take on in a similarity solution, then the solution ahead of the shock is said to be compatible with a similarity solution.

If the medium ahead of the shock is characterized by a function $G_{+}(x)$ and if it is compatible with a similarity solution, then it must be a similarity solution. We begin the proof of this statement by observing that due to the fact that $R$ is continuous across the shock we may replace the variables $p, w$ and $\varrho$ in Eqs. (6.15) through (6.21) by the variables 
$P, W$ and $N$ respectively. Since the variables $P_{-}, W_{-}, N_{-}, \varphi_{-}, \psi_{-}, \mathscr{R}_{-}$ are to describe a similarity solution, they are constant, that is independent of $r$ on the shock as is $V_{-}$.

If we define

$$
\begin{aligned}
& \beta=\frac{P_{-}}{W_{+}}=\frac{p_{-}}{w_{+}}, \\
& \alpha=\frac{P_{+}}{W_{+}}=\frac{p_{+}}{w_{+}}=\alpha\left(x_{+}\right), \\
& \gamma=\frac{W_{-}}{P_{-}}=\frac{w_{-}}{p_{-}}=\gamma\left(x_{-}\right)
\end{aligned}
$$

then Eq. (6.21) implies that $\beta=\beta\left(x_{+}, x_{-}\right)$and given $x_{+}$and $x_{-}$both $\alpha$ and $\beta$ are known.

Eq. (6.17) with $V_{-}$constant then determines the constant $x_{+}$and hence $W_{+}, P_{+}$and $N_{+}$. Eqs. (6.18), (6.20), and (6.21) enable us to determine $\dot{\mathscr{R}}_{+}, \varphi_{+}$and $\psi_{+}$as constants. $\mathscr{\mathscr { R }}_{+}$vanishes since $R_{+}=R_{-}\left(r, z_{1}\right)=r \mathscr{R}_{-}\left(z_{1}\right)$. Hence if the region behind the shock is described by a similarity solution, then the $Y^{A}$ of Eq. (5.7) vanish. Further it follows from the constancy of $P_{+}$and $x_{+}$that $K(r) r^{2}=k_{0}$ a constant. Hence the unique solution of equations (5.7) subject to the initial conditions derived from the jump relations is given by a similarity solution. It is of course assumed that the shock is such that

$$
\left(\mathscr{R}+\mathscr{R}^{*}\right)\left(V^{2}-a^{2}\right) \neq 0 .
$$

If the solution ahead of the shock is not characterized by a function $G_{+}(x)$, but one of the quantities $\alpha, \beta, \varphi_{+}$or $\psi_{+}$is constant along the shock with a similarity solution behind it, then each of them is independent of $r$. This statement is an immediate consequence of Eqs. (6.15) through (6.21).

\section{Strong Shocks}

In this section we shall assume that the medium behind the shock is characterized by the function

$$
G(x)=\frac{4}{x}
$$

and discuss the jump conditions and the similarity differential equations that obtain in this case. Eq. (8.1) is equivalent to the statement that

behind the shock and that

$$
w=3 p
$$

$$
W_{-}=3 P_{-}
$$


on the shock. Eq. (8.2) is the equation of state of a photon gas and that of a relativisitic gas at high temperature (cf. [5]). It is therefore reasonable to expect it to hold in the region immediately behind a very strong shock. We shall however in this section assume that it holds throughout the region of space-time described by a similarity solution.

It follows from Eq. (8.1) and Eqs. (1.14) and (1.25) that

$$
L(x)=x^{3}
$$

and that

$$
a^{2}=\frac{1}{3}=a_{-}^{2} .
$$

For such a medium Eqs. (6.16) through (6.21) become

$$
\begin{gathered}
\frac{\varrho_{-}^{2}}{\varrho_{+}^{2}}=\frac{N_{-}^{2}}{N_{+}^{2}}=\frac{4 \beta(3 \beta+\alpha)}{(1+\beta)(1+\alpha)}, \\
V_{+}^{2}=\frac{(\beta-\alpha)(3 \beta+\alpha)}{(3 \beta-1)(1+\beta)}=\mathrm{e}^{2 \psi_{+}-2 \varphi_{+}} z_{1}^{2}, \\
V_{-}^{2}=\frac{(\beta-\alpha)(1+\beta)}{(3 \beta-1)(3 \beta+\alpha)}=\mathrm{e}^{2 \psi_{1}-2 \varphi_{1}} z_{1}^{2}, \\
A^{2}=\frac{(\beta-\alpha)(3 \beta-1)}{(1+\beta)(3 \beta+\alpha)}, \\
\frac{-\mathrm{e}^{\psi--} \varphi_{+} z_{1}^{2} \mathscr{R}_{1}^{\prime}}{\mathscr{R}_{1}+z_{1} \mathscr{R}_{1}^{\prime}}=\frac{A+\tanh \omega_{+}}{1+A \tanh \omega_{+}}, \\
\mathrm{e}^{2 \varphi_{-}-2 \varphi_{+}}=\frac{(3 \beta+\alpha)(1+\alpha)}{4 \beta(1+\beta)}, \\
\mathrm{e}^{2 \psi--2 \psi+}=\frac{(1+\beta)(1+\alpha)}{4 \beta(3 \beta+\alpha)}
\end{gathered}
$$

respectively, where we have made use of the fact that there is a similarity solution in the - region. Thus the quantities with the subscript - are evaluated from the similarity solution at $z=z_{1}$.

It then follows from Eqs. (8.5) and (8.8) that

where

$$
V_{-}^{2}-a_{-}^{2}=V_{-}^{2}-\frac{1}{3}=\frac{-6\left(\beta-\beta_{1}\right)\left(\beta-\beta_{2}\right)}{(3 \beta-1)(9 \beta+\alpha)}
$$

$$
\begin{aligned}
& 2 \beta_{1}=1-\alpha+\sqrt{(1-\alpha)^{2}-\frac{4}{3} \alpha}, \\
& 2 \beta_{2}=1-\alpha-\sqrt{\left(1-\alpha^{2}\right)-\frac{4}{3} \alpha} \leqq 2 \beta_{1} .
\end{aligned}
$$


When $\alpha=1 / 3$ or $\alpha=3$, we have $\beta_{1}=\beta_{2}=\frac{1-\alpha}{2}$. From the definition of $\alpha$ (cf. Eqs. (7.1)) it is reasonable to restrict $\alpha$ so that

$$
\alpha \leqq \frac{1}{3}
$$

for this is equivalent to the condition that

Hence $\beta_{1}$ and $\beta_{2}$ are real.

$$
w_{+}-3 p_{+} \geqq 0 \text {. }
$$

We have from Eqs. (8.5) and (8.7) that

$$
V_{+}^{2}-1=\frac{(1+\alpha)(1-\alpha-2 \beta)}{(3 \beta-1)(1+\beta)} \leqq 0 .
$$

The last inequality arises from the requirement that the velocity of the shock relative to the material ahead of it be less than the velocity of light in this material. Hence we must restrict $\beta$ so that

$$
\frac{1-\alpha-2 \beta}{3 \beta-1} \leqq 0
$$

We observe that

$$
3 \beta-1=\frac{w_{-}}{w_{+}}-1 \geqq 0
$$

when $w_{-} \geqq w_{+}$as will be the case for most materials.

If we now require that

$$
\beta>\beta_{1}
$$

we will have

$$
V_{-}^{2}-a_{-}^{2}<0
$$

and

$$
V_{+}^{2}-1<0 .
$$

We shall assume the inequality (8.13) in the subsequent discussion.

The equations which describe the similarity solution in the region behind the shock when Eq. (8.1) holds are readily obtained from the discussion of Section 4. We have

$$
\begin{aligned}
P & =\frac{K_{0} \mathscr{R}^{2}}{x^{4}} \\
W+P & =\frac{4 K_{0} \mathscr{R}^{2}}{x^{4}} \\
\mathrm{e}^{-\varphi} & =\frac{B \mathscr{R}^{2}}{x^{3}} \\
\mathrm{e}^{\varphi} & =A \sqrt{z} x,
\end{aligned}
$$


where $A$ and $B$ are constants each of which may be taken to be one by using a scale transformation. When this is done Eqs. (4.22) and (4.23) become

$$
\ddot{\mathscr{R}}+\dot{\mathscr{R}}+\left(\frac{\dot{\mathscr{R}}}{2 \mathscr{R}}-\frac{\dot{x}}{x}\right)(3 \mathscr{R}+4 \dot{\mathscr{R}})=0
$$

and

$$
\frac{1}{2} \mathscr{R}^{4}+2 x^{4} z \frac{\dot{\mathscr{R}}}{\mathscr{R}}-\frac{\dot{x}}{x}\left(3 z x^{4}-\mathscr{R}^{4}\right)=4 x^{2} K_{0}
$$

respectively.

The last two equations have a first integral given by

$$
\frac{2 K_{0} \mathscr{R}^{2}}{x^{4}}\left(3+\frac{4 \dot{\mathscr{R}}}{\mathscr{R}}\right)=1+\frac{z}{x^{2}} \dot{\mathscr{R}}^{2}-\frac{\mathscr{R}^{4}}{x^{6}}(\mathscr{R}+\dot{\mathscr{R}})^{2} .
$$

This equation is obtained by evaluating and equating the two expressions given for $M$ in Eqs. (4.11) and (4.13).

We shall discuss in detail in a subsequent paper the solutions of Eqs. (8.15), (8.16), and (8.17) which satisfy certain regularity conditions. In the remainder of this paper we shall discuss a particular solution of these equations and two compatible solutions of the field equations to which it may be fitted. The particular solution we shall treat is given by the functions

$$
\begin{aligned}
x & =x_{0} z^{-1 / 2} \\
\mathscr{R} & =\mathscr{R}_{0} z^{-1 / 2}
\end{aligned}
$$

where the constants $x_{0}$ and $\mathscr{R}_{0}$ must be determined by the relations

$$
\begin{aligned}
x_{0}^{2} & =8 K_{0} \\
\mathscr{R}_{0}^{6} & =4 x_{0}^{6}=2048 K_{0}^{3}=\delta^{6} K_{0}^{3} \\
\delta & =2^{11 / 6} .
\end{aligned}
$$

For this solution we have

$$
\begin{aligned}
R & =r \mathscr{R}(z)=r^{1 / 2} t^{1 / 2} \mathscr{R}_{0} \\
\mathrm{e}^{\varphi} & =x_{0} \\
\mathrm{e}^{\varphi} & =\frac{x_{0}^{3}}{\mathscr{R}_{0}^{2}} t^{1 / 2} r^{-1 / 2} .
\end{aligned}
$$

Hence the line element is given by

$$
d s^{2}=x_{0}^{2} d t^{2}-\frac{t \mathscr{R}_{0}^{2}}{4}\left[\frac{d r^{2}}{r}+4 r d \Omega^{2}\right] .
$$


We may write this as

where

$$
d s^{2}=d \hat{t}^{2}-S^{2}(\hat{t})\left[d \hat{r}^{2}+\hat{r}^{2} d \Omega^{2}\right]
$$

$$
\begin{aligned}
\hat{t} & =x_{0} t \\
\hat{r} & =\frac{\mathscr{R}_{0}}{\sqrt{x_{0}}} r^{1 / 2} \\
S(\hat{t}) & =\hat{t}^{1 / 2} .
\end{aligned}
$$

Eq. (8.21) describes a Robertson-Walker space time with zero curvature for the space $\hat{t}=$ constant and the scale function given by Eq. (8.22). For this space time we have

$$
M=P=\frac{1}{3} W=\frac{K_{0} \mathscr{R}_{0}^{2}}{x_{0}^{4}} z=\frac{\delta^{2}}{64} z
$$

and

$$
p=\frac{1}{3} w=\frac{1}{8 \hat{t}^{2}} .
$$

For $t>0$ this Robertson-Walker space time is non-singular for all $r$ and $t$ and in particular in the region

$$
0 \leqq z \leqq z_{1}
$$

if the point $r=0, t=0$ is excluded.

It follows from Eqs. (8.18) to (8.20) that

$$
V^{2}=2 M
$$

and

$$
x^{2} M=\frac{K_{0} \delta^{2}}{8}=K_{1} .
$$

These two equations and the first of Eqs. (8.23) are of the form of Eqs. (4.36). It is thus evident that there is only one essential parameter in this solution.

\section{Compatible Static Solutions}

A solution of the Einstein field Eqs. (1.3) to (1.6), such that in the normalized comoving coordinate system introduced in Section 3 , it obeys the additional restrictions

$$
R_{t}=w_{t}=p_{t}=0
$$


is a static solution. We will now show that all static solutions compatible with a similarity solution are similarity solutions.

Eq. (9.1) implies that $R$ is a function of $r$ alone. For a compatible solution, it follows from the continuity of $R$ across the shock hypersurface, the hypersurface

$$
z=z_{1}
$$

we may then conclude that for a compatible static solution

$$
R=r \mathscr{R}_{1}=r \mathscr{R}\left(z_{1}\right) .
$$

Since $M$ is continuous and constant on the shock we must have for the static solution

$$
m=M_{1} R=r M_{1} \mathscr{R}_{1}
$$

along the shock. Since $m$ is a function of $r$ (cf. Eqs. (3.5) and (9.1)) this equation determines $m$ throughout the static region.

Eqs. (3.6) and (9.1) enable us to conclude that

$$
e^{-2 \psi}=\left(1-2 M_{1}\right) / \mathscr{R}_{1}^{2} .
$$

Thus $\psi$ is a constant throughout the static region and in particular on the shock. Thus it follows from the concluding remark of section 7 that $\alpha, \beta$, and $\varphi_{+}$are constants on the shock. Therefore

$$
w_{+}=\frac{P_{1}}{r^{2} \beta}=\frac{M_{1}}{\mathscr{R}_{1}^{2} r^{2}}=w(r) .
$$

The last of Eqs. (9.5) follow from Eqs. (9.3) and (3.4). We also have

$$
p_{+}=\alpha w_{+}=\frac{\alpha M_{1}}{\mathscr{R}_{1}^{2} r^{2}}=p(r),
$$

and thus

$$
p(r)=\alpha w(r)
$$

with $\alpha$ a constant. This equation may be interpreted as either the equation of state for the source of the static field or as determining the constant value of $x$, the inverse temperature, for a medium characterized by the function $G(x)$ by the equation

$$
x G(x)=\frac{w+p}{p}=\frac{\alpha+1}{\alpha} .
$$

Eq. (1.8) may be written as

$$
\varphi_{r}=\frac{-p_{r}}{w+p}=\frac{2 \alpha}{(1+\alpha) r} .
$$


Hence

$$
e^{\varphi}=g(t) r^{2 \alpha / 1+\alpha}
$$

and Eqs. (3.7) reads

$$
2\left(1-2 M_{1}\right) \alpha=M_{1}(1+\alpha)^{2} .
$$

That is, in the static medium

$$
P(r)=\alpha M_{1}=1-3 M_{1} \pm \sqrt{\left(4 M_{1}-1\right)\left(2 M_{1}-1\right)}=\alpha W(r)
$$

Since $e^{-2 \psi}$ and $M_{1}$ must be positive we must have

$$
0 \leqq 2 M_{1} \leqq 1 .
$$

The constant $\alpha$ will take on real values only if

$$
0<4 M_{1} \leqq 1 .
$$

The value of $\alpha$ determines the velocity of sound in the static medium if it satisfies the equation of state given above. In that case we must have

and

$$
\alpha \leqq 1
$$

$$
P=\alpha M_{1}=1-3 M_{1}-\sqrt{\left(4 M_{1}-1\right)\left(2 M_{1}-1\right)} .
$$

If we impose the condition that

$$
\alpha \leqq \frac{1}{3}
$$

then we find that we must have

$$
0 \leqq 14 M_{1} \leqq 3 .
$$

It follows from Eq. (9.7) and the requirement that $\varphi_{+}$is a constant that

$$
e^{\varphi}=C z^{n},
$$

where $C$ is a constant and

$$
n=\frac{2 \alpha}{1+\alpha} .
$$

Thus for every $M_{1}$ in the range given by the inequalities (9.8) or (9.10) we may determine a physically acceptable value of $\alpha$ by means of Eq. (9.9). Then the compatible static solution of the Einstein field equations is given by the line element

$$
d s^{2}=C^{2} z^{2 n} d t^{2}-\frac{\mathscr{R}_{1}^{2}}{1-2 M_{1}} d r^{2}-r^{2} \mathscr{R}_{1}^{2} d \Omega^{2}
$$


and is a similarity solution. Further, every compatible solution is a similarity solution with three parameters $\mathscr{R}_{1}, C$ and $M_{1}$. The first two of these may be removed by the scale transformation

$$
\begin{aligned}
& \hat{t}=C t, \\
& \hat{r}=\mathscr{R}_{1} r
\end{aligned}
$$

and thus there is only one essential parameter.

It will be shown in a subsequent paper that there is a two essential parameter family of similarity solutions satisfying the equation of state $w=3 p$ which are regular in the region

$$
0 \leqq z \leqq z_{1} .
$$

These parameters may be chosen so that the solution they determine may be fitted to the static similarity solution given above across a shock wave. That is, the $\varphi_{+}, \psi_{+}, M_{+}, P_{+}, W_{+}$determined above may be used to determine the corresponding quantities behind the shock. These in turn may be used as initial conditions for determining a solution of Eqs. (8.14) to (8.17). The solution so determined can be shown to be regular in the region given above.

The similarity solution discussed in Section 8 , which describes a Robertson-Walker metric, may be fitted to a compatible static solution for a particular value of the essential parameter $M_{1}$. That is, we may construct a solution of the field equations such that for the region behind the shock, the region

$$
0 \leqq z \leqq z_{1},
$$

we have the Robertson-Walker metric and for the region ahead of the shock, the region

$$
z>z_{1}
$$

we have the static solution given above. The solution so constructed will be nonsingular everywhere except at $r=0 \geqq t$ and the hypersurface $z=z_{1}$, the shock. As before we shall use subscript $-(+)$ to denote the value of a quantity from the region behind (ahead of) the shock at $z=z_{1}$. It remains to be shown that Eqs. (8.6) to (8.8) and Eqs. (8.11) and (8.12) may be satisfied.

We recall that for the Robertson-Walker solution we have

$$
P_{-}=M_{-}=\frac{V_{-}^{2}}{2}=\frac{K_{1}}{x_{-}^{2}}=\frac{1}{3} W_{-} .
$$

The continuity of $M$ implies that

$$
M_{+}=M_{-}=M_{1} .
$$


For the static solution we have Eq. (9.9) which must hold for $P_{+}$and

$$
W_{+}=M_{1} \text {. }
$$

When the above equations are used, Eqs. (8.8) become an equation for $M_{1}$, namely the equation

$$
2 M_{1}=\frac{M_{1}-P_{+}\left(M_{1}\right)}{3 M_{1}+P_{+}\left(M_{1}\right)}=V_{-}^{2} .
$$

where $P_{+}\left(M_{1}\right)$ is given by the right hand side of Eq. (9.9). That is, we must have

$$
M_{1}\left(2 M_{1}-1\right)\left(8 M_{1}^{2}+6 M_{1}-1\right)=0 .
$$

The value of $M_{1}$ satisfying this equation and consistent with the inequalities (9.10) is

$$
M_{1}=\frac{\sqrt{17}-3}{8} \text {. }
$$

Hence

$$
V_{-}^{2}=\frac{\sqrt{17}-3}{4}
$$

and we may use Eq. (8.7) to calculate

$$
V_{+}^{2}=\frac{\left(M_{1}-P_{+}\left(M_{1}\right)\right)\left(3 M_{1}+P_{+}\left(M_{1}\right)\right)}{4 M_{1}^{2}}=0.685
$$

since it follows from Eqs. (9.16) that

$$
P_{+}=0.0173 \text {. }
$$

If the scale is chosen so that Eqs. (8.20) and (9.13) hold, we may calculate $z_{1}$ from the relation

$$
M_{-}=M_{1}=\frac{\delta^{2}}{64} z_{1}=\frac{\sqrt{17}-3}{8} .
$$

The continuity of $R$ requires that

$$
\mathscr{R}_{1}=\frac{\mathscr{R}_{0}}{\sqrt{z_{1}}}
$$

and Eqs. (8.11) enables one to relate $x_{0}$ and $C$

$$
\frac{x_{0}}{C z_{1}^{n}}=\sqrt{\frac{\left(3 M_{1}+P_{+}\right)\left(M_{1}+P_{+}\right)}{8 M_{1}^{2}}}
$$

where $n$ is given by Eq. 9.12. Eq. (8.12) is satisfied as a consequence of Eq. (9.15) and the above equations. 
Not all similarity solutions may be fitted to the static solution across a shock. If the medium in the static region is characterized by a function $G_{+}(x)$ where $x$ is the reciprocal temperature, we may determine this quantity in the static region in terms of the parameter $M_{1}$ for in that region

$$
x G_{+}(x)=\frac{W}{P}+1=\frac{M_{1}}{P\left(M_{1}\right)}+1 .
$$

Eqs. (6.25) may be written as

$$
\frac{x_{+}^{2}}{x_{-}^{2}}=\frac{P_{-}^{2}\left(W_{+}+P_{+}\right)\left(W_{+}+P_{-}\right)}{P_{+}^{2}\left(W_{-}+P_{-}\right)\left(W_{-}+P_{+}\right)} .
$$

In this equation $P_{+}$and $W_{+}$are known as functions of the parameter $M_{1}$. However from the continuity of $M$ across the shock we may regard them as a function of $M_{-}$. Hence we may regard this equation as determining $x_{+}$as a function of $P_{-}, x_{-}$and $M_{-}$. This function of these variables must satisfy the equation

$$
x_{+} G_{+}\left(x_{+}\right)=\frac{M_{-}}{P_{+}\left(M_{-}\right)}+1,
$$

where $P_{+}\left(M_{-}\right)$is given by the right hand side of Eq. (9.9) with $M_{-}$ replacing $M_{1}$.

Eq. (6.23) may be written as

$$
V_{-}^{2}=\frac{\left(P_{-}-P_{+}\left(M_{-}\right)\right)\left(M_{-}+P_{-}\right)}{\left(W_{-}-M_{-}\right)\left(W_{-}+P_{+}\left(M_{-}\right)\right.} .
$$

Since $V_{-}$is determined as a function of $P_{-}, x_{-}$and $M_{-}$from the equations characterizing a similarity solution, Eq. (9.19) imposes another condition on the similarity solutions that may be fitted to a static one.

A similarity solution may be fitted to the static solution discussed in this section and characterized by a function $G(x)$ if and only if the similarity solution behind the shock contains a hypersurface $z=z_{1}$ on which Eqs. (9.18) and (9.19) are satisfied.

\section{Similarity Solutions for Dust}

If the source of the gravitational field is a fluid in which $p=0$, this medium is said to be incoherent matter or dust. For such matter, solutions of the Einstein field equations are well known and depend on the specification of the mass function $m(r, t)$ which in this case is a function of $r$ alone. The matter may be considered as obeying a caloric equation of 
state with any function $G(x)$ which is such that it equals unity when the temperature vanishes, that is, when $x$ the reciprocal temperature becomes infinite. In that case we have

$$
w=\varrho c^{2}
$$

or

$$
W=N \text {. }
$$

If a dust solution is to be compatible with a similarity solution, it too must be a similarity solution as follows from the discussion of Section 7. It is the purpose of this section to determine the subclass of dust solutions of the Einstein field equations which are similarity solutions and to fit one of them which represents collapsing dust to the explicit similarity solution, given in Section 8, across a shock hypersurface with the equation

$$
z=z_{1} .
$$

The determination of dust similarity solutions is carried out in the same manner as is the determination of a general dust solution. However, we need only deal with ordinary differential equations, Eqs. (4.10) through (4.16). Since $P=0$, we have from Eq. (4.16) that

and from (4.10) that

$$
e^{\varphi}=D
$$

$$
e^{\psi}=\frac{C}{W}=\frac{C}{N},
$$

where $C$ and $D$ are constants, which can be given any convenient values by a scale transformed.

Eq. (4.12) may also be integrated immediately and we obtain

$$
\mathscr{R} M=A C,
$$

where $A$ is a constant and $C$ is the constant introduced above. This equation is equivalent to the statement that

$$
m(r, t)=A C r .
$$

Thus the similarity dust solutions are that special class of the general dust solutions for which the mass function is linear and homogeneous.

Eqs. (4.13) and (4.11) become

$$
M=N\left(1+\frac{\dot{\mathscr{R}}}{\mathscr{R}}\right)=W\left(1+\frac{\dot{\mathscr{R}}}{\mathscr{R}}\right)=W\left(1-\frac{\dot{M}}{M}\right)
$$

and

$$
2 M=1+A^{2}\left(\frac{C^{2}}{D^{2}} z^{2} \frac{\dot{M}^{2}}{M^{4}}-1\right)
$$


respectively. If we recall that

$$
V=e^{\psi-\varphi} z=\frac{C}{D N} z=\frac{C}{D W} z
$$

and that

$$
\tanh \omega=e^{\psi-\varphi} \frac{R_{t}}{R_{r}}=-z V \frac{\dot{\mathscr{R}}}{\mathscr{R}+\dot{\mathscr{R}}}
$$

we may write the above equation as

$$
2 M=1+A^{2}\left(V^{2}\left(1-\frac{W}{M}\right)^{2}-1\right)=1-\frac{A^{2}}{\cosh ^{2} \omega} .
$$

The second form of this equation relates the velocity of the hypersurface $R=$ constant with respect to the fluid to the constant $A$ and the function $M$.

The solution to the ordinary differential Eq. (10.6) is given by

$$
B \pm \frac{D}{C z}=A X(M)=B \pm \frac{1}{W V}=B \pm \frac{1}{N V}
$$

where

$$
X(M)=\left\{\begin{array}{l}
\frac{-1}{\sqrt{M\left(1-A^{2}\right)}} \sqrt{\frac{2}{1-A^{2}}-\frac{1}{M}}+\frac{2}{\left(1-A^{2}\right)^{\frac{3}{2}}} \sin ^{-1}\left(\sqrt{\frac{1-A^{2}}{2 M}}\right), \\
A<1 \\
\frac{\sqrt{2} M^{-\frac{3}{2}},}{3} \quad A=1 \\
\frac{1}{\sqrt{M\left(A^{2}-1\right)}} \sqrt{\frac{2}{A^{2}-1}+\frac{1}{M}}-\frac{2}{\left(A^{2}-1\right)^{\frac{3}{2}}} \sinh ^{-1}\left(\sqrt{\frac{A^{2}-1}{2 M}}\right), \\
A>1 .
\end{array}\right.
$$

The solutions for which

$$
A \geqq 1, B>0, B-\frac{D}{C z}=A X(M)=B-\frac{1}{W V}=B-\frac{1}{N V}
$$

are non-singular for $t$ large and negative but for

$$
z=\frac{D}{C B}=z_{2},
$$

that is where

$$
B V N=1
$$

there is a singularity at which $M$ becomes infinite and hence $R=r \mathscr{R}=0$ as follows from Eq. (10.4). 
Let us now consider the problem of fitting a similarity dust solution across a shock which is a similarity hypersurface to another region of material characterized by a function $G$. The fitting conditions in the form (6.23) to (6.26) allow us to express $M_{+}, P_{+}, x_{+}$and $V_{+}$in terms of $M_{-}, P_{-}, x_{-}$and $V_{-}$where + refers to the dust region. Because $x_{+}\left(M_{-}, P_{-}, x_{-}, V_{-}\right)$vanishes there is a relation which the quantities on the minus side of the shock have to satisfy in order for this solution to be fittable to a dust solution. Because $P_{+}$vanishes and $N_{+}=W_{+}(6.23)$ and (6.25) may be solved for $W_{+}$and when the resulting expressions are equated we find

$$
\frac{1}{V_{-}^{2}}=\left(x_{-} G\left(x_{-}\right)-1\right)^{2}-x_{-}^{2} .
$$

There are two arbitrary essential constants in the dust solution and therefore Eq. (10.13) is the necessary and sufficient condition for a solution of the fitting equations to exist when the material on the + side is dust. Eq. (10.13) is the dust fitting compatability condition.

We now turn to the problem of fitting the explicit similarity solution discussed in section 8 to the collapsing dust one given above. We make use of the fitting conditions in the form (6.23) to (6.26). Let the shock occur at some value of $M, M_{s}$ say, which by continuity of $M$ satisfies

$$
M_{s}=M_{+}=M_{-} .
$$

The fitting compatability condition (10.13), with the aid of (8.25) and (8.26), becomes

$$
K_{1}=9 M_{s}-\frac{1}{2}
$$

Eq. (6.23) becomes

$$
W_{+}=M_{s} \frac{\left(18 M_{s}-1\right)}{\left(6 M_{s}+1\right)}
$$

and with the aid of this Eq. (6.24) is expressible as

$$
V_{+}^{2}=\frac{\left(6 M_{s}+1\right)^{2}}{32 M_{s}} .
$$

The condition that $V_{+}^{2}$ be less than unity may thus be written

$$
\frac{1}{18}<M_{s}<\frac{1}{2} \text {. }
$$

Eq. (10.7) may now be solved for $A$ and yields

$$
A^{2}=\frac{1}{4\left(18 M_{s}-1\right)}
$$


while (10.10) gives $B$ as

$$
B=4 \sqrt{\frac{2\left(6 M_{s}+1\right)}{18 M_{s}-1}}+A X\left(M_{s}\right),
$$

where (10.18) must be used for $A$. The inequality (10.17) guarantees that the two solutions determined through (10.14), (10.18), and (10.19), with a separating shock occuring at some $M_{s}$ on the indicated interval, are physically acceptable. Thus $M_{s}$ generates a one-parameter family of space-times such that in each case the collapsing dust solution described above is terminated by a strong shock at $M=M_{s}$. Behind this shock is a region described by the Robertson-Walker solution obtained in section 8 . Thus by introducing the shock hypersurface we obtain a solution of the Einstein field equations which is non-singular except at $r=0, t=0$ and on the shock hypersurface. The collapse of the dust is stopped by the shock which "converts" the dust into a medium for which $w=3 p$. Such a medium could be considered to be a photon gas. At $r=0, t>0$, that is, $z=0$, the space time is regular but the temperature, pressure and energy density vanish. Hence it may be physically unrealistic to say that the medium behind the shock obeys the equation of state $p=3 w$ for all $M$ in the range

$$
0 \leqq M \leqq M_{s}
$$

if it is to be regarded as composed of material particles. In a subsequent paper we shall examine the situation that arises when a similarity dust solution given above is struck by a shock wave behind which the medium is characterized by the function $G(x)$ given by Synge [5] for a relativistic gas of material particles. The dust case is of course covered by this function $G$ when the temperature vanishes and therefore the same kind of material is on both sides of the shock. We propose to determine whether there is a similarity solution behind the shock which is regular except at $r=0, t=0$.

\section{References}

1. Courant, R., Friedricho, K. O.: Supersonic flow and shock waves, p. 419. New York: Interscience Publishers 1948.

2. Neumann, J. von: Collected Works. Vol. VI, p. 219. Oxford: Pergamon Press 1963.

3. Taylor, G. I.: Proc. Roy. Soc. London A 201, 159 (1950).

4. Carrus, P. A., Fox, Phyllis, A., Haas, Felix, Kopal, Zdeněk: Astrophys. Jour. 113, 193 (1951); 113, 496 (1951). — Kopal, Zdeněk: Astrophys. Jour. 120, 159 (1954).

5. Synge, J.: The relativistic gas. Amsterdam: North-Holland Publishing 1957.

A. H. Taub and M. E. Cahill Mathematics Department University of California Berkeley, Cal. 94720, USA 